\title{
Do Orally Administered Immunoglobulin-Based Agents Represent the Future of IBD Therapeutics?
}

\author{
Jason M. Shapiro ${ }^{1,2} \cdot$ Basavaraj Kerur ${ }^{1,2}$
}

Received: 4 July 2015/Accepted: 6 July 2015/Published online: 17 July 2015

(C) Springer Science+Business Media New York 2015

Inflammatory bowel disease (IBD), commonly divided into Crohn's disease and ulcerative colitis, is a chronic, debilitating condition characterized by relapsing and remitting episodes of gastrointestinal inflammation. While the exact etiology of IBD is unknown, its pathogenesis has been attributed to numerous factors, including genetic predisposition, alterations in the gut microbiome, defects in the innate and adaptive immune system, and putative environmental exposures [1]. The majority of currently available therapies mostly alter immune responses rather than target-specific factors that may elicit an aberrant, gutspecific inflammatory cascade. Of these, the intestinal microbiome is an attractive candidate for such IBD-specific therapeutics, particularly since it is altered in patients with IBD. One such example of an IBD-specific dysbiosis is the observation that certain strains of pathogenic adherentinvasive Escherichia coli (LF82) are associated with persistent ileal inflammation in patients with Crohn's disease [2]. Combined with the observation that animal models of colitis require gut bacterial colonization in order to induce an inflammatory phenotype, the importance of the microbiome in IBD pathogenesis is emphasized.

In this issue of Digestive Diseases and Sciences, Henderson et al. assessed the effect of oral serum-derived bovine protein isolate (SBI) administration on downstream

Jason M. Shapiro

jshapiro@lifespan.org

1 Division of Pediatric Gastroenterology, Nutrition and Liver Diseases, Department of Pediatrics, Hasbro Children's Hospital/Rhode Island Hospital, 593 Eddy Street, Providence, RI 02903, USA

2 The Warren Alpert School of Medicine, Brown University, Providence, RI, USA mucosal inflammation in a microbiota-defined LF82/dextran sulfate sodium (DSS) mouse model of colitis [3]. SBI is a specifically formulated protein preparation that contains $>50 \%$ bovine immunoglobulin $\mathrm{G}$ (IgG), its use based on the observation that the immunoglobulins in SBI reduce bacterial antigen-associated intestinal inflammation via immune and steric exclusion mechanisms [4]. In the accompanying manuscript, the investigators reported reduced mucosal and systemic inflammation in the LF82/ DSS mouse model when administered SBI compared with controls treated with the unrelated protein, hydrolyzed collagen. Based on these results, the authors conclude that SBI-immunoglobulin binding of bacterial antigens in the intestinal lumen may inhibit the downstream activation of gut-specific inflammatory cascades that have been implicated in IBD pathogenesis.

The potential therapeutic effects of SBI are likely multifactorial. While early work focused on SBI's potential value as a protective nutrient that supports the physiologic digestive and absorptive properties of the intestinal tract, recent data support its ability to bind specific bacterial antigens that promote and sustain inflammation, reduce pro-inflammatory cytokine production, and improve intestinal barrier function. SBI is used clinically to treat conditions such as irritable bowel syndrome-diarrhea predominant (IBS-D), antibiotic-associated diarrhea, small intestinal bacterial overgrowth, and human immunodeficiency virus (HIV)-associated enteropathy [5]. A mechanism similar to that proposed for SBI was reported by Greenberg et al. [6], who demonstrated the successful use of colostrum-derived bovine immunoglobulin for the treatment of diarrhea caused by Cryptosporidium parvum infection in immunosuppressed patients with acquired immunodeficiency syndrome (AIDS). 
In general, the oral delivery of antibody-based therapeutics in human disease can be challenging due to the rapid proteolytic degradation that normally occurs in the gastrointestinal tract. Since immunoglobulins present in bovine colostrum are partially resistant to digestion by gastric and intestinally secreted proteolytic enzymes relative to the proteins usually ingested in the diet, bovinederived protein products are attractive candidates for such a therapeutic approach [7]. How these results from a DSS mouse model will translate into clinical IBD management, however, is difficult to predict. While previous studies have reported positive effects of SBI in the treatment of other enteropathies such as IBS-D and HIV-associated diarrhea, mouse models of colitis are, in general, simplistic representations of what is a complex genetic, immune-mediated disease process in humans. The altered Schaedler flora (ASF) is a community of eight bacterial species selected based on their dominance in the normal microflora of healthy mice. Administered to germ-free mice for control purposes, the ASF is far from a comprehensive representation of the diverse ecosystem that is the human microbiome. In addition, the LF82 mouse model, which incorporates only a single bacterial strain, is not representative of the complex dysbiosis associated with human IBD. Furthermore, while hydrolyzed collagen has a relatively low antigenicity profile, it has entirely different physicochemical properties compared with immunoglobulins. Whether a non-immune globulin to test whether SBI was a specific or non-specific antigen binder would have been a more meaningful control is worth consideration.

Despite the pitfalls inherent to any animal model of human disease, the proposed mechanism of action of SBI for IBD management has similarities to the currently accepted practice of using exclusive enteral nutrition (EEN) with polymeric formulas. Although not antibody based, the use of EEN also alters the intestinal flora, enhances barrier function, and decreases mucosal and systemic concentrations of inflammatory cytokines in patients with IBD [8]. Commonly used for both the induction and maintenance of remission in Crohn's disease, a meta-analysis of pediatric studies concluded that EEN was as effective as systemic corticosteroids in patients with active disease [9]. Although effective, the long-term use of EEN can be challenging. Whether or not the more targeted effects of immunoglobulin-based therapies such as SBI could be used as to augment or replace the immunomodulatory effects of EEN in IBD is provocative and worth further exploration.

Conceptually, the approach suggested by the authors offers a promising new avenue for research into therapeutic orally administered antibodies in the treatment of IBD. These agents could theoretically deliver specific oral antibodies directly to the inflamed gut while avoiding systemic exposure, thus mitigating a potential systemic allergic response. Furthermore, oral antibodies such as SBI and colostrum-derived bovine immunoglobulin might be modified to improve their bacterial specificity for, or engineered as chimeric antibodies or as newer biologic agents that block specific pro-inflammatory pathways. One such example is AVX-470 [10], a bovine-derived polyclonal anti-tumor necrosis factor alpha (TNF- $\alpha$ ) antibody. In a mouse model of colitis, a trial of orally delivered AVX 470 was not only well tolerated but also localized almost entirely to the intestinal tract, with minimal systemic exposure. AVX-470 was resistant to digestive proteases, and active antibody was recovered in stool samples after oral administration of enteric-coated capsules [10]. A more attractive therapeutic approach, however, would be the targeting of pro-inflammatory bacterial antigens rather than reactive cytokines, such as TNF- $\alpha$. While antibody responses to bacterial antigens such as flagellin and OmpC have been incorporated into diagnostic and prognostic algorithms of IBD, specific proteins that may be targeted for therapeutic purposes are yet to be identified. As intestinal proteolytic stability is an inherent property of bovine colostral antibodies, they may be uniquely suited to the development of such gut or bacterial-targeted therapeutics in the future.

In conclusion, treating IBD is a difficult balance of controlling active symptoms and preventing long-term complications of aggressive disease while being mindful of the potential serious side effects inherent to immunemodulating therapies. The majority of currently available medications indicated for IBD management suppress the immune system and, as a result, are associated with numerous adverse side effects associated with immune suppression, including the development of malignancies. While disease onset and progression appear to be associated with alterations in the intestinal microbiome, these observations are yet to be clinically translated into effective therapies. This well-conceived animal study presents preliminary data suggesting that an orally delivered, immunoglobulin-derived agent may represent a novel, safe, and reasonable therapeutic approach for the management of IBD.

\section{References}

1. Abraham C, Cho JH. Inflammatory bowel disease. $N$ Engl J Med. 2009;361:2066-2078.

2. Darfeuille-Michaud A, Boudeau J, Bulois P, et al. High prevalence of adherent-invasive Escherichia coli associated with ileal mucosa in Crohn's disease. Gastroenterology. 2004;127:412-507.

3. Henderson A, Brand MW, Darling RJ, et al. Attenuation of colitis by serum-derived bovine immunoglobulin/protein isolate in a 
defined microbiota mouse model. Dig Dis Sci. (Epub ahead of print). doi:10.1007/s10620-015-3726-5.

4. Detzel CJ, Horgan A, Henderson AL, et al. Bovine immunoglobulin/protein isolate binds pro-inflammatory bacterial compounds and prevents immune activation in an intestinal coculture model. PLoS One. 2015;10:e120278.

5. Petschow BW, Blikslager AT, Weaver EM, et al. Bovine immunoglobulin protein isolates for the nutritional management of enteropathy. World J Gastroenterol. 2014;20:11713-11726.

6. Greenberg PD, Cello JP. Treatment of severe diarrhea caused by Cryptosporidium parvum with oral bovine immunoglobulin concentrate in patients with AIDS. J Acquir Immune Defic Syndr Hum Retrovirol. 1996;13:348-354.
7. Roos N, Mahe S, Benamouzig R, et al. $15 \mathrm{~N}$-labeled immunoglobulins from bovine colostrum are partially resistant to digestion in human intestine. J Nutr. 1995;125:1238-1244.

8. Day AS, Lopez RN. Exclusive enteral nutrition in children with Crohn's disease. World J Gastroenterol. 2015;21:6809-6816.

9. Heuschkel RB, Menache CC, Megerian JT, et al. Enteral nutrition and corticosteroids in the treatment of acute Crohn's disease in children. J Pediatr Gastroenterol Nutr. 2000;31:8-15.

10. Bhol KC, Tracey DE, Lemos BR, et al. AVX-470: a novel oral anti-TNF antibody with therapeutic potential in inflammatory bowel disease. Inflamm Bowel Dis. 2013;19:2273-2280. 